\title{
The Geometric Cauchy Problem for the Hyperbolic Hessian One Equation ${ }^{1}$
}

\author{
Antonio Martínez and Francisco Milán
}

Departamento de Geometría y Topología, Universidad de Granada, E-18071 Granada, Spain.

e-mail: amartine@ugr.es, milan@ugr.es

Keywords: Cauchy problem, hyperbolic hessian one equation, improper affine spheres. 2000 Mathematics Subject Classification: 53A15.

\begin{abstract}
We solve the problem of finding all indefinite improper affine spheres passing through a given regular curve of $\mathbb{R}^{3}$ with a prescribed affine co-normal vector field along this curve. We prove the problem is well-posed when the initial data are non-characteristic and show that uniqueness of the solution can fail at characteristic directions. As application we classify the indefinite improper affine spheres admitting a geodesic planar curve.
\end{abstract}

\section{Introduction}

Differential geometry of surfaces and partial differential equations (PDEs) have a strong link by means of which both theories benefit mutually. One of the problems that is currently experiencing a great development is the geometric version of the classical Cauchy problem for a second order PDE. It can be formulated as follows:

Let $\mathcal{S}$ be a class of surfaces in a Riemannian 3 -manifold $\mathcal{M}$ and denote by $\beta(s)$ a regular curve in $\mathcal{M}$. If $\mathcal{P}(s)$ is a distribution of oriented planes along $\beta$ in the tangent bundle of $\mathcal{M}$, one can pose the following

Geometric Cauchy problem: Find the surfaces in $\mathcal{S}$ that pass through $\beta(s)$ and whose tangent plane distribution along $\beta(s)$ is precisely $\mathcal{P}(s)$.

The problem is well-posed if there is a unique solution which depends continuously of the data.

This geometric problem has been considered for many classes of surfaces whose underlying PDEs associated are either elliptic or hyperbolic. There have been many papers concerning to the elliptic case, see $[1,3,4,7,8,9]$. In all of them the classes

\footnotetext{
${ }^{1}$ Research partially supported by Ministerio de Educación y Ciencia Grant No. MTM2013-43970-P and Junta de Andalucía Grant No. FQM-325.
} 
of surfaces considered admit Weierstrass' type representations in terms of holomorphic data and so, the surfaces are real analytic and it is necessary to prescribed analytic initial data (one has well-posedness problems under the analytic regularity assumption but the problem is $\mathcal{C}^{\infty}$ ill-posed).

In contrast to the elliptic case, the problem when the underlying PDE associated is hyperbolic, is in many respects, far from complete. If one considers lesser regular data $\mathcal{C}^{\infty}$ or even worst, many problems arise. Actually, these classes of surfaces can not be analytic and gluing procedures may create unexpected situations.

One of biggest contributions from geometry to PDEs theory is Monge Ampére type equations which model interesting geometric aspects related to the curvature. Among the most outstanding hyperbolic Monge-Ampére equation we have the hyperbolic Hessian one equation

$$
\operatorname{det} \nabla^{2} f+1=0, \quad \text { in } \Omega \subseteq \mathbb{R}^{2}
$$

which arise in affine differential geometry as the equation of indefinite improper affine spheres, that is, surfaces in $\mathbb{R}^{3}$ with a Lorentzian affine metric and whose affine normals

at all points are parallel. This totally non linear PDE appears also strongly linked to flat surfaces in $\mathbb{S}^{3}$, see $[2,6]$ as well as to area distances in computer vision (distances that are useful in matching two images of the same object from different points of view), see $[17]$.

Recently, in [15], the second author studied the geometric Cauchy problem for indefinite improper affine spheres by considering analytic and non-characteristic initial data. In this paper, we extend these results in two directions, first, as in [2], we consider regular initial data without any analytic assumption and second, we also analyze the problem with characteristic initial data and show the differences with the well-posed non-characteristic problem.

The work is organized as follows. In section 2 we briefly review indefinite improper affine spheres and remind the Blaschke's representation for this class of surfaces.

In Section 3 we discuss some examples that help us to understand the results in the two following sections. Section 4 is devoted to solve the geometric Cauchy problem for indefinite improper affine spheres when the initial data are non-characteristic. In Section 5 we extend our study to the characteristic case and show that uniqueness of solution can fail at characteristic directions. Finally, in Section 6 we classify the indefinite improper affine spheres admitting a geodesic planar curve.

\section{Blaschke's representation}

Consider $\psi: \Sigma \longrightarrow \mathbb{R}^{3}$ an indefinite improper affine sphere, that is, an immersion with constant affine normal $\xi$ and Lorentzian affine metric $h$. Then, see [10, 16], up to an equiaffine transformation, one has $\xi=(0,0,1)$ and $\psi$ can be locally seen as the graph of a solution $f(x, y)$ of (1.1).

In such case, the indefinite affine metric $h$ of $\psi$ is given by

$$
h=f_{x x} d x^{2}+2 f_{x y} d x d y+f_{y y} d y^{2},
$$

the affine co-normal $N$ is

$$
N=\left(-f_{x},-f_{y}, 1\right)
$$


and (1.1) is equivalent to

$$
\sqrt{|\operatorname{det}(h)|}=\left[\psi_{x}, \psi_{y}, \xi\right]=-\left[N_{x}, N_{y}, N\right],
$$

that is, the volume element of $h$ coincides with the determinant $[., ., \xi]$.

We also observe that $h=-\langle d N, d \psi\rangle$ and $N$ is determined by

$$
\langle N, \xi\rangle=1, \quad\langle N, d \psi\rangle=0,
$$

with the standard inner product $\langle$,$\rangle in \mathbb{R}^{3}$. Moreover, from (1.1), (2.1) and (2.2), one can obtain

$$
\Delta_{h} N=0,
$$

where $\Delta_{h}$ is the Laplace-Beltrami operator associated to $h$.

Actually, see [5], if we take asymptotic parameters $(u, v)$ for $h$, then from $(2.3)$ and (2.4) we have

$$
h=2 \rho d u d v, \quad \rho=\left\langle N, \psi_{u v}\right\rangle=\left[\psi_{u}, \psi_{v}, \xi\right]=-\left[N, N_{u}, N_{v}\right]>0
$$

and

$$
\xi=\frac{1}{\rho} N_{v} \times N_{u}, \quad N=\frac{1}{\rho} \psi_{u} \times \psi_{v}
$$

where by $\times$ we denote the cross product in $\mathbb{R}^{3}$. Also, we get

$$
\psi_{u}=N \times N_{u}, \quad \psi_{v}=N_{v} \times N, \quad N_{u}=\psi_{u} \times \xi, \quad N_{v}=\xi \times \psi_{v}
$$

and

$$
\psi_{u v}=\rho \xi, \quad N_{u v}=0 .
$$

Hence, there exist, globally, two regular curves $a(u)$ and $b(v)$ in $\mathbb{R}^{2}$ defined by

$$
N-\xi \times \psi=(2 a, 1), \quad N+\xi \times \psi=(2 b, 1),
$$

such that

$$
N(u, v)=(a(u)+b(v), 1), \quad \xi \times \psi(u, v)=(b(v)-a(u), 0)
$$

and

$$
\rho(u, v)=-\operatorname{det}\left(a^{\prime}(u), b^{\prime}(v)\right)>0 .
$$

Remark 1. Observe that from (2.7) and (2.9), if $\Sigma$ is simply connected, a global asymptotic immersion $(u, v): \Sigma \longrightarrow \mathbb{R}^{2}$ can be given as

$$
u=\int \sqrt{<d a, d a>}, \quad v=\int \sqrt{<d b, d b>} .
$$


Moreover, from (2.6) and (2.8), if we write $a=\left(a_{1}, a_{2}\right)$ and $b=\left(b_{1}, b_{2}\right)$, then the coordinates of $\psi$ are

$$
\begin{aligned}
& \psi_{1}=b_{2}-a_{2} \\
& \psi_{2}=a_{1}-b_{1} \\
& \psi_{3}=b_{1} a_{2}-b_{2} a_{1}+\int a_{1} d a_{2}-a_{2} d a_{1}+\int b_{2} d b_{1}-b_{1} d b_{2} .
\end{aligned}
$$

Remark 2. From (2.8), the asymptotic curve $\beta(u)=\psi\left(u, v_{0}\right)$, determines the curve $a(u)$ and the affine co-normal $\eta(u)=N\left(u, v_{0}\right)$ along it, but not the curve $b(v)$. So, in the characteristic case,

$$
0=h\left(\beta^{\prime}(u), \beta^{\prime}(u)\right)=-\left\langle\eta^{\prime}(u), \beta^{\prime}(u)\right\rangle,
$$

there exist many improper affine spheres containing the curve $\beta$, with a prescribed affine co-normal $\eta$ along $\beta$.

\section{Ruled examples}

It is well-known that, up to an equiaffine transformation, a ruled improper affine sphere $\psi$ is the graph of a solution of (1.1) given by

$$
f(x, y)=x y+2 g(x),
$$

for a function $g: I \longrightarrow \mathbb{R}$, see $[5,11,12]$.

In this case, from (2.1), the affine metric is given by

$$
h=2 g^{\prime \prime}(x) d x^{2}+2 d x d y=2 d u d v,
$$

with $u=x$ and $v=g^{\prime}(x)+y$. Thus, from (2.2), the immersion $\psi$ and its affine co-normal $N$ can be parameterized, respectively, as

$$
\psi(u, v)=\left(u, v-g^{\prime}(u), u v-u g^{\prime}(u)+2 g(u)\right)
$$

and

$$
N(u, v)=\left(-g^{\prime}(u)-v,-u, 1\right)=(a(u)+b(v), 1) .
$$

From (2.8) and (2.11),

$$
a(u)=-\left(g^{\prime}(u), u\right), \quad b(v)=-(v, 0),
$$

satisfy $\operatorname{det}\left(a^{\prime}(u), b^{\prime}(v)\right)=-1$, for all $(u, v) \in I \times \mathbb{R}$.

In particular, the regular curve $\beta: I \longrightarrow \mathbb{R}^{3}$ defined by

$$
\beta(s)=(s, 0,2 g(s))
$$

is contained in $\psi(I \times \mathbb{R})$, with affine co-normal $\eta(s)=\left(-2 g^{\prime}(s),-s, 1\right)$ along it. Moreover, they can be obtained as

$$
\beta(s)=\psi(u(s), v(s)), \quad \eta(s)=N(u(s), v(s))=(\widetilde{a}(s)+\widetilde{b}(s), 1),
$$


with

$$
u(s)=s, \quad v(s)=g^{\prime}(s), \quad \widetilde{a}(s)=a(s), \quad \widetilde{b}(s)=b(v(s)) .
$$

Note that $v \in \mathbb{R}$ is a global parameter for the regular curve $b(v)=-(v, 0)$ but $s$, with $v(s)=g^{\prime}(s)$, maybe not.

Of course, when $g^{\prime}(s)$ is a diffeomorphism onto its image, with inverse $s(v)$, we can recover $a$ and $b$ as $a(u)=\widetilde{a}(u)$ and $b(v)=\widetilde{b}(s(v))$.

However, see Remark 2, if we take $g(s)=0$, then $\beta(s)=\psi(s, 0)$ is an asymptotic curve and $\{\beta, \eta\}$ does not determine, uniquely, the pair $\{\psi, N\}$.

Similarly, for $g(s)=s^{3}$, we have $v(s)=3 s^{2}$ and $v^{\prime}(0)=0$, that is, $\beta(\mathbb{R})$ is contained on the upper side $\psi(\mathbb{R} \times[0,+\infty[)$ and is tangent to the asymptotic curve $\psi(\mathbb{R} \times\{0\})$ at $\beta(0)=\psi(0,0)$.

Hence, around $(0,0)$, we have many improper affine spheres containing the curve $\beta$, with affine co-normal $\eta$ along it. For instance, $\widehat{\psi}:]-\varepsilon, \varepsilon[\times]-\varepsilon, \varepsilon\left[\longrightarrow \mathbb{R}^{3}\right.$ given by (2.11), with $\widehat{a}(u)=-\left(g^{\prime}(u), u\right)=-\left(3 u^{2}, u\right)$ and

$$
\widehat{b}(v)= \begin{cases}-(v, 0), & v \geq 0 \\ -\left(v, \exp \left(-k / v^{2}\right)\right), & v<0\end{cases}
$$

for some real constants $k>0$ and $\varepsilon>0$, such that $\operatorname{det}\left(\widehat{a}^{\prime}(u), \widehat{b}^{\prime}(v)\right) \neq 0$, see Figure 1 .
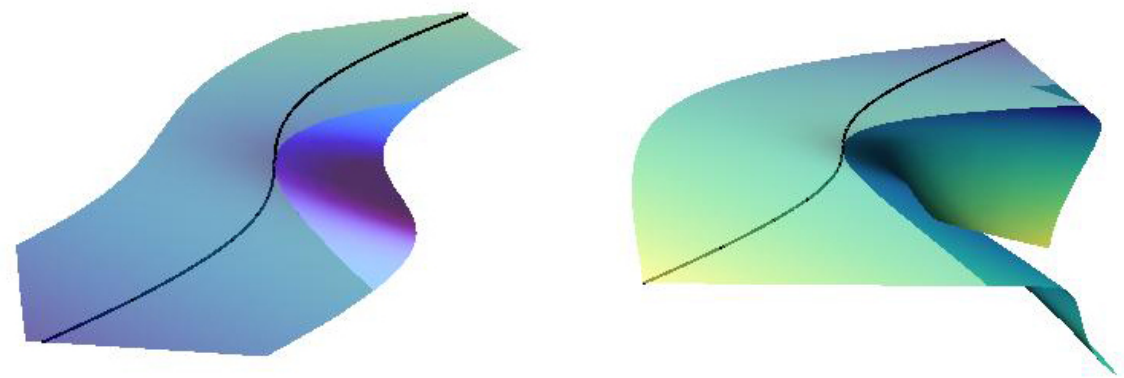

Figure 1: Indefinite improper affine spheres containing $\beta(s)=\left(s, 0,2 s^{3}\right)$ with $\eta(s)=$ $\left(-6 s^{2},-s, 1\right)$.

\section{The non-characteristic Case}

The Blaschke's representation let us study the geometric Cauchy problem of finding all the indefinite improper affine spheres $\psi$ containing a regular curve $\beta: I \longrightarrow \mathbb{R}^{3}$ with a prescribed affine co-normal $\eta: I \longrightarrow \mathbb{R}^{3}$ along it. However, the above examples show that the situation is different of the analytic case studied in $[1,5,15]$, specially when $\langle d \beta, d \eta\rangle$ vanishes.

Hence, see (2.4) and Remark 2 , we will say that $\{\beta, \eta\}$ is a non-characteristic admissible pair if satisfies the conditions

$$
\langle\xi, \eta(s)\rangle=1, \quad\left\langle\beta^{\prime}(s), \eta(s)\right\rangle=0,
$$


with $\xi=(0,0,1)$ and

$$
\lambda(s)=\left\langle\beta^{\prime \prime}(s), \eta(s)\right\rangle=-\left\langle\beta^{\prime}(s), \eta^{\prime}(s)\right\rangle \neq 0, \quad \forall s \in I .
$$

In this case, we can obtain a solution $\psi$, such that $\beta$ never is tangent to its asymptotic (also known as characteristic) curves. Moreover, the solution is unique around $\beta(I)$, that is, any two solutions agree on an open set containing $\beta(I)$. Actually, we have

Theorem 4.1. Let $\beta, \eta: I \longrightarrow \mathbb{R}^{3}$ be a non-characteristic admissible pair of curves. Then, in a neighborhood of $\beta(I)$, there exists a unique indefinite improper affine sphere $\psi$ containing $\beta(I)$ with affine co-normal $\eta(s)$ at $\beta(s)$ for all $s \in I$.

Proof. Assume there exists an immersion $\psi$ with affine co-normal $N$, solution of the above geometric Cauchy problem, then the curves $a$ and $b$ given by (2.7) satisfy

$$
4 \operatorname{det}(d a, d b)=2[d N, \xi \times d \psi, \xi]=2[d \eta, \xi \times d \beta, \xi]=2\langle d \beta, d \eta)\rangle \neq 0,
$$

along $\beta$. Hence, see (2.5) and (2.9), any parameters $u$ of $a$ and $v$ of $b$ are asymptotic parameters around $\beta(I)$, where, by the inverse function theorem, we have

$$
\beta(s)=\psi(u(s), v(s)), \quad \eta(s)=N(u(s), v(s))
$$

and

$$
2 \rho(u(s), v(s)) u^{\prime}(s) v^{\prime}(s)=-\left\langle\beta^{\prime}(s), \eta^{\prime}(s)\right\rangle .
$$

So, from (4.2) and (4.3), $u(s)$ and $v(s)$ are diffeomorphisms onto their images, with inverses $s(u)$ and $s(v)$, respectively.

From (2.7) and (2.11), if we take the curves $\widetilde{a}(s)$ and $\widetilde{b}(s)$ defined by

$$
(\eta-\xi \times \beta)(s)=(2 \widetilde{a}(s), 1), \quad(\eta+\xi \times \beta)(s)=(2 \widetilde{b}(s), 1),
$$

then,

$$
a(u)=\widetilde{a}(s(u)), \quad b(v)=\widetilde{b}(s(v)),
$$

and $\psi$ is uniquely determined by $\{\beta, \eta\}$, which proves the uniqueness of $\psi$ around $\beta(I)$.

For the existence, we consider the above curves $\widetilde{a}(s)$ and $\widetilde{b}(s)$. Now, from $(4.1),(4.2)$ and (4.4), we have

$$
4 \operatorname{det}\left(\widetilde{a}^{\prime}(s), \widetilde{b}^{\prime}(s)\right)=2\left[\eta^{\prime}(s), \xi \times \beta^{\prime}(s), \xi\right]=2\left\langle\beta^{\prime}(s), \eta^{\prime}(s)\right\rangle \neq 0 .
$$

Thus, from (2.7), (2.9), (2.11) and (4.4), for any diffeomorphisms $u(s)$ and $v(s)$, with images $u(I)$ and $v(I)$, the regular curves $a(u)$ and $b(v)$ given by (4.5) satisfy

$$
\operatorname{det}\left(a^{\prime}(u), b^{\prime}(v)\right)=\operatorname{det}\left(\widetilde{a}^{\prime}(s), \widetilde{b}^{\prime}(s)\right) s^{\prime}(u) s^{\prime}(v) \neq 0
$$

and provide an indefinite improper affine sphere $\psi: u(I) \times v(I) \longrightarrow \mathbb{R}^{3}$, containing $\beta(I)$ with affine co-normal $\eta$ along it.

Remark 3. It is clear that Theorem 4.1 extends Theorem 3.1 in [15], since from the above construction the solution for a non-characteristic analytic admissible pair must be analytic around the curve. 


\section{The characteristic Case}

Again from Remark 2, we know that a pair $\{\beta, \eta\}$ generates many indefinite improper affine sphere $\psi$, when $\langle d \beta, d \eta\rangle$ vanishes identically. Hence, we will assume that (4.1) holds and that $\left\langle\beta^{\prime}(s), \eta^{\prime}(s)\right\rangle$ only vanishes at isolated points.

Even under this assumption, the examples of Section 3, see also Figure 1, show that we should not expect uniqueness of the solution of the above geometric Cauchy problem.

The key point in the proof of Theorem 4.1 is that the following relation holds

$$
\left\langle\beta^{\prime}(s), \eta^{\prime}(s)\right\rangle=-2 \rho(u(s), v(s)) u^{\prime}(s) v^{\prime}(s)
$$

which suggests divide this section in two classes of characteristic points:

\subsection{Characteristic points with sign.}

Definition 5.1. Let $\beta, \eta: I \longrightarrow \mathbb{R}^{3}$ be an admissible pair of curves, we say that $s_{0} \in I$ is a characteristic point with sign if $\left\langle\beta^{\prime}\left(s_{0}\right), \eta^{\prime}\left(s_{0}\right)\right\rangle=0$ and $\left\langle\beta^{\prime}(s), \eta^{\prime}(s)\right\rangle$ does not change sign around $s_{0}$. Equivalently, $u^{\prime}\left(s_{0}\right) v^{\prime}\left(s_{0}\right)=0$ and both $u^{\prime}(s)$ and $v^{\prime}(s)$ do not change sign around $s_{0}$, since $\left(u^{\prime}\left(s_{0}\right), v^{\prime}\left(s_{0}\right)\right) \neq(0,0)$ by the regularity of $\beta(s)=\psi(u(s), v(s))$.

In this case, $u(s)$ and $v(s)$ are strictly monotone because their derivates do not change sign and only vanish at isolated points. In particular, they are diffeomorphisms onto their images and, analogously to the proof of Theorem 4.1, we can prove the uniqueness. Actually, we have:

Proposition 5.2. Let $\beta, \eta: I \longrightarrow \mathbb{R}^{3}$ be an admissible pair of curves such that all their characteristic points are isolated and have sign. Then, $\{\beta, \eta\}$ determines at most an indefinite improper affine sphere $\psi$ containing $\beta(I)$ with affine co-normal $\eta$ along $\beta$.

In order to prove existence, we need some additional conditions, which are trivial for non-characteristic points.

In fact, from (2.11) and (4.5), the images of the curves $\widetilde{a}(s)$ and $\widetilde{b}(s)$ given by (4.4) must be regular, since they will be the projection of the asymptotic curves of $\psi$. So, from (5.1), we can deduce:

Proposition 5.3. Let $\{\beta, \eta\}$ be an admissible pair of curves such that all their characteristic points are isolated and have sign. If the traces of the curves $\widetilde{a}(I)$ and $\widetilde{b}(I)$ are regular, then there exists a solution of the corresponding geometric Cauchy problem, if and only if, for every $s_{0} \in I$ the limit

$$
\lim _{s \rightarrow s_{0}} \frac{\left\langle\beta^{\prime}(s), \eta^{\prime}(s)\right\rangle}{u^{\prime}(s) v^{\prime}(s)}
$$

exists and is non-zero, for some parameterizations $u(s)$ of $\widetilde{a}(I)$ and $v(s)$ of $\widetilde{b}(I)$.

Note that the condition (5.2) cannot hold if $\left\langle\beta^{\prime}(s), \eta^{\prime}(s)\right\rangle$ changes sign, since $u^{\prime}(s)$ and $v^{\prime}(s)$ are always non-negative, up to the orientation. 


\subsection{Characteristic points without sign.}

Definition 5.4. Let $\beta, \eta: I \longrightarrow \mathbb{R}^{3}$ be an admissible pair of curves, we say that $s_{0} \in I$ is a characteristic point without sign if $\left\langle\beta^{\prime}\left(s_{0}\right), \eta^{\prime}\left(s_{0}\right)\right\rangle=0$ and $\left\langle\beta^{\prime}(s), \eta^{\prime}(s)\right\rangle$ changes sign around $s_{0}$.

Equivalently, from (5.1), if $\beta(s)=\psi(u(s), v(s))$, then either $u^{\prime}\left(s_{0}\right)=0, v^{\prime}\left(s_{0}\right) \neq 0$ and $u^{\prime}(s)$ changes sign around $s_{0}$ or the similar case for $(v, u)$. So, we can assume, without loss of generality, that $u(s)$ has a local maximum $u_{0}$ at $s_{0}$ and $v^{\prime}\left(s_{0}\right) \neq 0$.

This means that $\beta$ is tangent to the asymptotic curve $\psi\left(u_{0}, v\right)$ and around $\beta\left(s_{0}\right)=$ $\psi\left(u_{0}, v_{0}\right)$ is contained in the left side $\left.\left.\psi(] u_{0}-\varepsilon, u_{0}\right] \times\right] v_{0}-\varepsilon, v_{0}+\varepsilon[)$, for some $\varepsilon>0$.

As consequence, from the construction in Theorem 4.1, $\{\beta, \eta\}$ determines the curves $a(u)$ in $\left.] u_{0}-\varepsilon, u_{0}\right]$ and $b(v)$ in $] v_{0}-\varepsilon, v_{0}+\varepsilon\left[\right.$. That is, around $\beta\left(s_{0}\right)$, we have many solutions $\widehat{\psi}$ of the geometric Cauchy problem, which can be determined for (2.11), by taking $\widehat{a}$ and $\widehat{b}$ in the following way

$$
\widehat{a}(u)= \begin{cases}a(u), & \left.u \in] u_{0}-\varepsilon, u_{0}\right] \\ \bar{a}(u), & u \in\left[u_{0}, u_{0}+\varepsilon[\right.\end{cases}
$$

and $\widehat{b}(v)=b(v)$ in $] v_{0}-\varepsilon, v_{0}+\varepsilon\left[\right.$, for any curve $\bar{a}(u)$, different from $a(u)$, for $u \neq u_{0}$, such that $\operatorname{det}\left(\bar{a}^{\prime}(u), b^{\prime}(v)\right) \neq 0$ and such that $\widehat{a}$ is regular.

However, we can recover the improper affine sphere $\psi$ in terms of $\{\beta, \eta\}$ for the left side of $\left(u_{0}, v_{0}\right)$ and we have uniqueness on an open set with the characteristic point in its boundary. More specifically, as in Proposition 5.2 we can deduce the following general uniqueness result:

Theorem 5.5. Let $\beta, \eta: I \longrightarrow \mathbb{R}^{3}$ be an admissible pair of curves such that all their characteristic points are isolated. Then, two solutions to the corresponding geometric Cauchy problem agree on a domain which contains $\beta(I)$ except its characteristic points without sign.

For the existence around a characteristic point without $\operatorname{sign} s_{0} \in I$, note that the local maximum $u_{0}$ at $s_{0}$ implies that the traces of the curve $\widetilde{a}(s)=a(u(s))$ in $\left.] s_{0}-\delta, s_{0}\right]$ and $\left[s_{0}, s_{0}+\delta[\right.$ agree, for some $\delta>0$.

Conversely, if the curve $\widetilde{a}(s)$ given by (4.4) satisfies $\widetilde{a}\left(s_{-}\right)=\widetilde{a}\left(s_{+}\right)$, where $s_{-}=s_{0}-s$ and $s_{+}=s_{0}+s$ for all $\left.s \in\right] 0, \delta\left[\right.$, then we can take diffeomorphisms $u\left(s_{-}\right)$and $u\left(s_{+}\right)$onto ]$u_{0}-\varepsilon, u_{0}\left[\right.$, such that $\widetilde{a}\left(s_{-}(u)\right)=\widetilde{a}\left(s_{+}(u)\right)$. Thus, the solutions of the non-characteristic Cauchy problem in $] s_{0}-\delta, s_{0}[$ and $] s_{0}, s_{0}+\delta\left[\right.$ are given by the same $a(u)=\widetilde{a}\left(s_{-}(u)\right)=$ $\widetilde{a}\left(s_{+}(u)\right)$ in $] u_{0}-\varepsilon, u_{0}[$ and $b(v)=\widetilde{b}(s(v))$ in $] v_{0}-\varepsilon, v_{0}+\varepsilon[$.

Consequently, in a neighborhood of $s_{0}$, we get a well-defined solution $\psi$ by extending $a(u)$ to $] u_{0}-\varepsilon, u_{0}+\varepsilon[$ and from Proposition 5.3 we can conclude the following general existence result:

Theorem 5.6. Let $\{\beta, \eta\}$ be an admissible pair of curves such that all their characteristic points $s_{0}$ are isolated. If the traces of the curves $\widetilde{a}$ and $\widetilde{b}$ given by (4.4) are regular, then $\{\beta, \eta\}$ generates an indefinite improper affine sphere $\psi$ if, and only if the following conditions hold

i) The limit (5.2) exists and is non-zero, when $s_{0}$ has sign. 
ii) The curve $\widetilde{a}$ or $\widetilde{b}$ that is singular at $s_{0}$ has the same image on $] s_{0}-\delta, s_{0}[$ and ]$s_{0}, s_{0}+\delta\left[\right.$, for some $\delta>0$, when $\left\langle\beta^{\prime}(s), \eta^{\prime}(s)\right\rangle$ changes sign around $s_{0}$.

\section{Improper affine spheres with a planar geodesic}

Let $\mathcal{P}$ be a plane in $\mathbb{R}^{3}$ and $\beta: I \longrightarrow \mathcal{P}$ be a geodesic of an indefinite improper affine sphere $\psi$ with affine normal $\xi=(0,0,1)$. It is not a restriction to assume that the origin is in $\mathcal{P}$.

We will distinguish two cases:

CASE I: $\xi \in \mathcal{P}$.

In this case, up to an equiaffine transformation, we can assume that $\mathcal{P} \equiv\{y=0\}$ and so,

$$
\beta(s)=(x(s), 0, z(s)), \quad \eta(s)=\left(\eta_{1}(s), \eta_{2}(s), 1\right)
$$

satisfy

$$
0=<\beta^{\prime}(s), \eta(s)>=x^{\prime}(s) \eta_{1}(s)+z^{\prime}(s) .
$$

As $\beta$ is regular, this expression implies that $x^{\prime}(s) \neq 0$ and $x$ is also a parameter of $\beta$. That is, we can consider $x(s)=s$ and write

$$
\beta(s)=(s, 0, g(s)), \quad \eta(s)=\left(-g^{\prime}(s), \eta_{2}(s), 1\right) .
$$

From Corollary 4.4 in [15] and Remark 3, as $\beta$ is a geodesic of $\psi$ we have

$$
0=\left[\beta^{\prime}, \beta^{\prime \prime}, \xi\right]=\left[\eta, \eta^{\prime}, \eta^{\prime \prime}\right]
$$

or equivalently,

$$
c_{1} \eta_{1}+c_{2} \eta_{2}=c_{3},
$$

for some constants $c_{1}, c_{2}$ and $c_{3}$, such that $\left(c_{1}, c_{2}\right) \neq(0,0)$.

From (2.9) and (4.4), we obtain

$$
\begin{aligned}
& 2 \widetilde{a}(s)=\left(\eta_{1}(s), \eta_{2}(s)-s\right), \quad 2 \widetilde{b}(s)=\left(\eta_{1}(s), \eta_{2}(s)+s\right), \\
& 2 \widetilde{a}^{\prime}(s)=\left(\eta_{1}^{\prime}(s), \eta_{2}^{\prime}(s)-1\right), \quad 2 \widetilde{b}^{\prime}(s)=\left(\eta_{1}^{\prime}(s), \eta_{2}^{\prime}(s)+1\right),
\end{aligned}
$$

and

$$
4 \operatorname{det}\left(\widetilde{a}^{\prime}(s), \widetilde{b}^{\prime}(s)\right)=2 \eta_{1}^{\prime}(s)=-2 g^{\prime \prime}(s)=-4 \rho(s) u^{\prime}(s) v^{\prime}(s) .
$$

If $c_{2}=0$ in (6.3), then $\eta_{1}$ is a constant and $u^{\prime} v^{\prime} \equiv 0$. Thus, $\beta$ is an asymptotic straight line and either $\widetilde{a}$ or $\widetilde{b}$ is a constant curve. In this case, it is easy to check that $(\log (\rho))_{u v}=0$ for any asymptotic parameters $(u, v)$ around $\beta$. That is, $\psi$ has zero affine Gauss curvature and it must be a ruled surface in a neighborhood of $\beta$, see $[11,12]$.

If $c_{2} \neq 0$, then $\eta_{2}=-k g^{\prime}+c$ for some constants $k$ and $c$ and we have that

$$
2 \widetilde{a}(s)=\left(-g^{\prime}(s),-k g^{\prime}(s)+c-s\right), \quad 2 \widetilde{b}(s)=\left(-g^{\prime}(s),-k g^{\prime}(s)+c+s\right),
$$


are regular curves. Thus, we can take $u(s)=s=v(s)$ and from (4.5),

$$
\begin{aligned}
& 2 a(u)=\left(-g^{\prime}(u),-k g^{\prime}(u)+c-u\right), \\
& 2 b(v)=\left(-g^{\prime}(v),-k g^{\prime}(v)+c+v\right), \\
& h=\rho(u, v) d u d v=-\left(g^{\prime \prime}(u)+g^{\prime \prime}(v)\right) d u d v .
\end{aligned}
$$

From the above expressions and (2.11), we conclude that $\beta$ must be a convex curve and up to an equiaffine transformation, $\psi$ can be recover as follows, see Figure 2:

$$
\psi(u, v)=\left(\frac{u+v}{2}, \frac{g^{\prime}(v)-g^{\prime}(u)}{2}, \frac{\left(g^{\prime}(u)-g^{\prime}(v)\right)(v-u)}{4}+\frac{g(v)+g(u)}{2}\right) .
$$
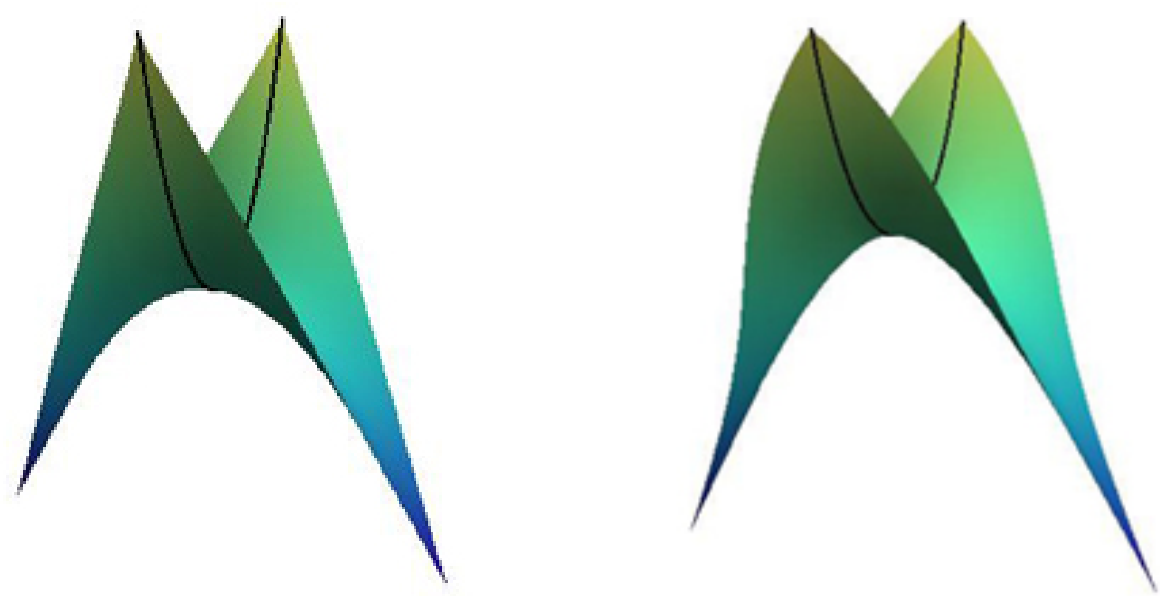

Figure 2: Indefinite improper affine spheres with geodesics $\beta(s)=\left(s, 0, s^{2}\right)$ and $\beta(s)=$ $(s, 0, \cosh (s))$, respectively.

\section{CASE II: $\xi \notin \mathcal{P}$.}

In this case, up to an equiaffine transformation, we may assume that $\mathcal{P} \equiv\{z=0\}$. Moreover, as $\beta=(x(s), y(s), 0)$ is a geodesic of $\psi$, we have from Corollary 4.4 in [15] that,

$$
\begin{gathered}
x^{\prime} y^{\prime \prime}-y^{\prime} x^{\prime \prime}=\left[\beta^{\prime}, \beta^{\prime \prime}, \xi\right]=\left[\eta, \eta^{\prime}, \eta^{\prime \prime}\right], \\
<\beta^{\prime}, \eta>=0, \quad<\beta^{\prime \prime}, \eta>=m,
\end{gathered}
$$

for some constant $m$. Consequently, either $\beta$ is an asymptotic straight line if $m=0$ and, discussing as in the above case, $\psi$ is ruled in a neighborhood of $\beta$, or $\beta$ is a convex curve if $m \neq 0$.

When $\beta$ is convex, we can assume that $\beta$ is parameterized by its affine arc-length, see $[13,18]$, that is,

$$
\left[\beta^{\prime}(s), \beta^{\prime \prime}(s), \xi\right]=1, \quad \forall s \in I,
$$


which implies that

$$
\beta^{\prime \prime \prime}(s)+\kappa(s) \beta^{\prime}(s)=0,
$$

where $\kappa(s)=\left[\beta^{\prime \prime}(s), \beta^{\prime \prime \prime}(s), \xi\right]$ is the affine curvature of the planar curve $\beta$.

Moreover, from (4.1) and (6.7), $\eta$ is determined by $\beta$ as follows:

$$
\eta=\beta^{\prime} \times\left(\beta^{\prime \prime}-m \xi\right) .
$$

Thus, from (6.6), (6.8) and (6.9), we obtain $1=m^{2} \kappa(s)$, for all $s \in I$ and the affine curvature is a positive constant.

Then, up to an equiaffine transformation, $\beta$ is contained in a circle of radius $m^{3 / 2}$ and we may write

$$
\begin{aligned}
& \beta(s)=\left(m^{3 / 2} \cos \left(\frac{s}{m}\right), m^{3 / 2} \sin \left(\frac{s}{m}\right), 0\right), \quad \forall s \in I, \\
& \eta(s)=\left(-m^{3 / 2} \cos \left(\frac{s}{m}\right),-m^{3 / 2} \sin \left(\frac{s}{m}\right), 1\right) .
\end{aligned}
$$

Now, from (2.11) and (4.4), we obtain regular curves

$$
\begin{aligned}
2 \widetilde{a}(s) & =m^{3 / 2}\left(-\cos \left(\frac{s}{m}\right)+\sin \left(\frac{s}{m}\right),-\cos \left(\frac{s}{m}\right)-\sin \left(\frac{s}{m}\right)\right), \\
2 \widetilde{b}(s) & =m^{3 / 2}\left(-\cos \left(\frac{s}{m}\right)-\sin \left(\frac{s}{m}\right),+\cos \left(\frac{s}{m}\right)-\sin \left(\frac{s}{m}\right)\right)
\end{aligned}
$$

and the indefinite improper affine sphere $\psi$ containing the circle $\beta$ as a geodesic can be parameterized as follows,

$$
\begin{aligned}
& \psi_{1}(u, v)=\frac{m^{3 / 2}}{2}\left(\cos \left(\frac{v}{m}\right)+\cos \left(\frac{u}{m}\right)+\sin \left(\frac{u}{m}\right)-\sin \left(\frac{v}{m}\right)\right), \\
& \psi_{2}(u, v)=\frac{m^{3 / 2}}{2}\left(\cos \left(\frac{v}{m}\right)-\cos \left(\frac{u}{m}\right)+\sin \left(\frac{u}{m}\right)+\sin \left(\frac{v}{m}\right)\right), \\
& \psi_{3}(u, v)=\frac{m^{3}}{2} \cos \left(\frac{u-v}{m}\right)+\frac{m^{2}}{2} u-\frac{m^{2}}{2} v-\frac{m^{3}}{2} .
\end{aligned}
$$

Here, from (2.5), the affine metric is

$$
h=\frac{m}{4} \cos \left(\frac{u-v}{m}\right) d u d v
$$

and $\psi$ has singularities when $u-v=m(2 n+1) \pi / 2$, for some $n \in \mathbb{Z}$, see Figure 3 and [14]. 


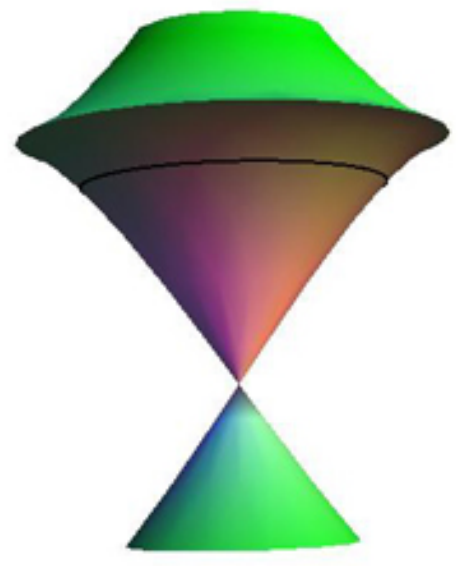

Figure 3: Indefinite improper affine sphere with geodesic $\beta(s)=(\cos (s), \sin (s), 0)$.

All the above discussion can be summarized in the following result:

Theorem 6.1. If $\beta: I \longrightarrow \mathcal{P}$ is geodesic of an indefinite improper affine sphere $\psi$ with affine normal $\xi=(0,0,1)$. Then in a neighborhood of $\beta$ and, up to an equiaffine transformation, either

i) $\beta$ is an straight line and $\psi$ is a ruled surface, or

ii) $\beta(s)=(s, 0, g(s))$ is convex and $\psi$ can be given given as in (6.5), or

iii) $\psi$ is rotational and it can be given as in (6.12).

Note that the symmetries in the above examples are consequence of the extension of Theorem 5.1 in [15] that follows from Theorems 4.1 and 5.5,

Theorem 6.2. Any symmetry of an admissible pair, such that all their characteristic points are isolated and have sign, induces the corresponding symmetry of the indefinite improper affine sphere generated by it.

\section{References}

[1] J.A. Aledo, R.M.B. Chaves and J.A. Gálvez, The Cauchy Problem for Improper Affine Spheres and the Hessian One Equation, Trans. Am. Math. Soc. 359 (2007), 4183-4208.

[2] J.A. Aledo, J.A. Gálvez and P. Mira, A D'Alembert formula for flat surfaces in the 3-sphere, J. Geom. Anal. 19 (2009), 211-232.

[3] J.A. Aledo, A. Martínez and F. Milán, The affine Cauchy problem, J. Math. Anal. Appl. 351 (2009), 70-83.

[4] L.J. Alías, R.M.B. Chaves and P. Mira, Björling problem for maximal surfaces in Lorentz-Minkowski space, Math. Proc. Camb. Philos. Soc. 134 (2003), 289-316. 
[5] W. Blaschke, Vorlesungen über Differentialgeometrie II. Affine Differentialgeometrie. Springer, 1923.

[6] L. Bianchi, Sulle superficie a curvatura nulla in geometria elliptica, Ann. Mat. Pura Appl. 24 (1896), 93-129.

[7] J.A. Gálvez and P. Mira, Dense solutions to the Cauchy problem for minimal surfaces, Bull. Braz. Math. Soc. 35 (2004), 287-294.

[8] J.A. Gálvez and P. Mira, The Cauchy problem for the Liouville equation and Bryant surfaces, Adv. Math. 195 (2005), 456-490.

[9] J.A. Gálvez and P. Mira, Embedded isolated singularities of flat surfaces in Hyperbolic 3-space, Calc. Var. Partial Dif. 24 (2005), 239-260.

[10] A.M. Li, U. Simon and G. Zhao, Global affine differential geometry of hypersurfaces, Walter de Gruyter 1993.

[11] M.A. Magid and P. Ryan, Flat affine spheres in $\mathbb{R}^{3}$, Geom. Dedicata 33(3) (1990), $277-288$.

[12] A. Martínez and F. Milán, On affine-maximal ruled surfaces, Math. Z. 208 (1991), 635-644.

[13] A. Martínez, F. Milán and I. Val, Teoría afín de curvas planas, Gaceta de la RSME 16 (2013), 271-281.

[14] F. Milán, Singularities of improper affine maps and their Hessian equation, J. Math. Anal. Appl. 405 (2013), 183-190.

[15] F. Milán, The Cauchy problem for indefinite improper affine spheres and their Hessian equation, Adv. Math. 251 (2014), 22-34.

[16] K. Nomizu and T. Sasaki, Affine differential geometry, Cambridge University Press 1994.

[17] M. Niethammer, S. Betelu, G. Sapiro, A. Tannenbaum and P.J. Giblin, Area-based medial axis of planar curves, Internat. J. Comput. Vision, 60 (2004), 203-224

[18] B. Su, Affine differential geometry, Science Press, Beijing 1983. 